\title{
Comparación de la seguridad al deslizamiento de seis presas españolas. Criterios de diseño empleados frente al estado del arte actual.
}

\section{Comparison of the sliding safety of six Spanish dams. Design criteria used versus current state of the art.}

\author{
Julián Alonso Pérez $^{(*)}$, Luis Romera Rodríguez ${ }^{(*)}$
}

\section{RESUMEN}

Se ha desarrollado y aplicado un método simplificado para reevaluar de forma más precisa la seguridad al deslizamiento en seis presas de gravedad y contrafuertes en base a los resultados de modelos lineales numéricos, verificando su validez mediante análisis no lineales y medidas experimentales. Se han llevado a cabo sondeos con recuperación de testigo cercanos al tacón aguas arriba para inspeccionar posibles agrietamientos en las interfases hormigón-roca y se han instrumentado con extensómetros para analizar los datos de aperturas. Los resultados obtenidos, demuestran que los criterios de diseño utilizados en los años 50, en algunos casos no cubren el margen de incertidumbre esperado, respaldando así la necesidad de revisar las grandes presas españolas actualizando los análisis tal como rezan las Normas Técnicas de Seguridad de Presas que recientemente ha aprobado el Gobierno.

Palabras clave: Seguridad al deslizamiento; subpresión; grieta; extensómetros; criterios de diseño.

\section{ABSTRACT}

A simplified method has been developed and applied to reevaluate accurately sliding safety in six gravity and buttresses dams based on the results of numerical linear models. The method has been validated by nonlinear analysis and experimental measurements. Rotary boreholes have been drilled, with core recovery, close to the upstream face, to study the current state of the concrete-rock interface and a possible presence of cracks. Extensometers have been installed and cracks opening data has been analyzed. The results obtained show that the design criteria used in the 1950s, in some cases do not cover the expected margin of uncertainty. The need of reassessmenting of Spanish large dams is supported, updating data as Technical Standards for Dam Safety recently approved by the Government of Spain require.

Keywords: Sliding safety; uplift; crack, extensometer, Design criteria.

(*) Ing. de Caminos. Responsable de los Servicios Técnicos de la generación hidroeléctrica de Endesa (Enel Green Power) en España y miembro vocal del Comité Nacional Español de Grandes Presas -SPANCOLD-. (España).

(**) Dr. Ingeniero Industrial. Profesor titular en el Área de Mecánica de los medios continuos y teoría de estructuras en la ETS Ingenieros de Caminos de la Universidad de La Coruña. (España).

Persona de contacto/Corresponding author: julianalberto.alonso@gmail.com (J. Alonso)

ORCID: http://orcid.org/oooo-0oo2-0764-033X (J. Alonso); http://orcid.org/oooo-0001-7756-5024 (L. Romera)

Cómo citar este artículo/Citation: Julián Alonso Pérez, Luis Romera Rodríguez (2021). Comparación de la seguridad al deslizamiento de seis presas españolas. Criterios de diseño empleados frente al estado del arte actual. Informes de la Construcción, 73(562): e392. https://doi. org/10.3989/ic.76918

Copyright: (C) 2021 CSIC. Este es un artículo de acceso abierto distribuido bajo los términos de la licencia de uso y distribución Creative Commons Reconocimiento 4.0 Internacional (CC BY 4.0). 


\section{INTRODUCCIÓN}

La Instrucción de Grandes Presas de 1967 (1) nacida por la rotura de la Presa de Vega de Tera tenía vigencia en las presas ya construidas sólo en lo referente a la necesidad de disponer Normas de Explotacion y un Archivo Técnico. Las presas construidas tras su entrada en vigor fueron diseñadas bajo estos criterios, pero en las presas anteriores, el proyectista utilizaba los coeficientes de seguridad que circulaban en versiones previas a la Instrucción, o a su propio juicio por falta de legislación específica. El Reglamento de 1996 (2) tenía retroactividad en todas las presas ya construidas en materia de análisis del riesgo potencial frente a la rotura pero en el resto de aspectos técnicos de diseño los titulares privados no tenían obligación de su aplicación. En 2003 el Comité Nacional Español de Grandes Presas publica la guía técnica número 2 (3) donde establece y actualiza los criterios para el diseño de presas, que son referencia en el ámbito especializado, y define coeficientes de seguridad y periodos de retorno de las avenidas en base a la clasificación de presas.

Estas legislaciones y recomendaciones demandan de alguna manera su regulación para analizar el parque existente bajo un mismo criterio. En 2008 con la modificación del Reglamento del Dominio Público Hidráulico (4) el Ministerio dedica un nuevo título relativo a la seguridad de presas, embalses y balsas; y anuncia, entre otros aspectos, la redacción de unas futuras Normas Técnicas de Seguridad de Presas que unifiquen la regulación con aplicación a todas las presas existentes. Desde entonces, se han elaborado 4 versiones de borradores que culminan en el Proyecto de Real Decreto (5) para la aprobación de las Normas Técnicas con la reciente publicación por parte del Ministerio en 2019 para participación pública. En consecuencia, será obligatorio el análisis de la seguridad del sistema presa-embalse comprendiendo aspectos estructurales, hidrológicos, sísmicos, y todos aquellos relativos al correcto funcionamiento y explotación. Esta normativa retroactiva supondrá un importante esfuerzo para los concesionarios o titulares privados, teniendo en cuenta el gran número de presas de titularidad privada construidas en la mitad del siglo XX.

Para analizar la justificación de este requerimiento y en base a la repercusión y consecuencias que tiene la revisión estructural, se plantea este estudio analizando la seguridad al deslizamiento de seis presas y que presentamos en los siguientes apartados. El análisis se centra en el modo de fallo al deslizamiento por ser la principal causa de fallo de presas de gravedad según el ICOLD (6), utilizando métodos numéricos y adicionalmente mediante una serie de trabajos experimentales que los sustentan.

El deslizamiento de presas siempre ha preocupado a la comunidad ingenieril, investigando y aprendiendo de fallos. En 1895 se produjo la catástrofe de la presa francesa de Bouzey (7) quizá la primera con consecuencias catastróficas bien documentada. Unas 200 personas murieron por la inundación. La comunidad científica se volcó en el estudio de las causas y la principal conclusión fue, que existía un factor, realmente importante, no tenido en cuenta hasta ese momento en los cálculos. La presa era estable, pero las filtraciones bajo la presa habían producido un empuje vertical hacia arriba, que se empezó a llamar subpresión, o presión intersticial que explicaba las razones del desastre. La distribución lineal de tensiones verticales supuesta bajo la presa no era real, ya que la subpresión introducía otras cargas verticales de sentido opuesto.
No fue hasta el último tercio del siglo XX, cuando el "Bureau of Reclamation”, estableció la metodología de cálculo para el proyecto de presas de gravedad, "Design of Small Dams, 1965” (8) y “Design of Gravity Dams, 1976”(9), donde se contempla la subpresión, debida al agrietamiento del contacto en el tacón de aguas arriba, y una distribución de empujes superior a la ley teórica triangular.

Con estos criterios se han diseñado las presas de gravedad, y de contrafuertes, especialmente hasta los años 70 (10), adoptando una ley lineal de tensiones en su base, aunque ya existía la inquietud generalizada por determinar la distribución real de tensiones verticales en el contacto. En 1971, Zienkiewicz, inicio la aplicación del método de los elementos finitos en ingeniería civil publicando, "The Finite Element Method in Engineering Science”(11). Esta revolucionaria metodología, puso de manifiesto la posible aparición de tracciones y agrietamientos en el pie de aguas arriba de este tipo de presas.

En años posteriores la ingeniería clásica en España incorpora la posibilidad de la existencia de un agrietamiento y la importancia de la subpresión en la estabilidad al deslizamiento (12), (13). En 1999 se presentan los resultados a este problema planteado en el Benchmark del ICOLD (14) de Denver utilizando modelos de elementos finitos, simulando la interfase del cimiento presa-roca y considerando la mecánica de la fractura en elementos cementosos. En 2004, el ingeniero italiano Giovanni Ruggeri lidera dos grupos de trabajo en el ICOLD europeo que culmina con la publicación de dos excelentes Informes Técnicos relativos a la subpresión (15) actualizando el estado del arte respecto a la estabilidad al deslizamiento (16).

Son muchos los estudios que ahondan en el tema. Destaca por su desarrollo, el análisis de Cai (17) utilizando modelos no lineales en el hormigón de una presa para calcular los agrietamientos, y sus efectos en la seguridad al deslizamiento en comparación con la mecánica clásica. Casi simultáneamente, los profesores Barpi y Valente del Politécnico de Turín (18) hacen un análisis similar pero considerando la mecánica de la fractura en la formación y progresión de la grieta. Ambas referencias ponen en evidencia la incertidumbre en el campo de la estabilidad, pero adolecen de contrastes experimentales.

Moftakhar y Ghafonry (19) en 2011, analizan una presa de gravedad, aplicando los criterios de las tres Agencias norteamericanas de seguridad de presas y comparando sus resultados con los de un modelo de elementos finitos con distribuciones no lineales de tensiones. Los resultados indican que los cálculos numéricos llevan a determinar una longitud de grieta mayor que la obtenida con los criterios de las agencias norteamericanas, destacando la importancia del posible agrietamiento en la estabilidad al deslizamiento, y poniendo de manifiesto que el margen de seguridad no es todo lo amplio que se esperaba. En un trabajo de 2013, McKay y López (20) proponen para el cálculo tensodeformacional de una presa de gravedad curva, un modelo de elementos finitos, introduciendo la presión intersticial tanto en el cuerpo de presa como en el cimiento y, por tanto, en el contacto presa-terreno. En la interfase presa-cimiento, se aborda la subpresión y el avance del agrietamiento mediante elementos no lineales y un método iterativo de avance.

En 2015 Krounis (21) estudia la sobrevaloración de los coeficientes de seguridad al deslizamiento calculados por la 
metodología clásica del equilibrio límite mediante modelos numéricos. Combina dos efectos fundamentales en los resultados, por un lado, el comportamiento frágil de la superficie de contacto roca-hormigón y por otro la distribución superficial de la cohesión a lo largo de dicho contacto, asumiendo diferentes valores espaciales según distribuciones estadísticas. Se concluye que ha de considerarse la posible existencia de puntos débiles en el contacto que reducen considerablemente la resistencia y los coeficientes de seguridad. La autora cuestiona los valores límite para los coeficientes de seguridad que definen las diferentes normas de presas y pone en duda que se cubra siempre la incertidumbre, abogando por la introducción de la metodología probabilista para la revisión de la seguridad.

En España Escuder y sus colaboradores han venido desde 2012 (22) aportando un nuevo enfoque probabilista al problema de la estabilidad al deslizamiento (23) creando una corriente a la hora de abordar la gestión de presas para una buena gobernanza (24). En 2016 Morales, Escuder, Altarejos y Serrano (25) aplican el análisis de riesgos al modo de fallo de deslizamiento de una presa de gravedad española, planteando una separación entre la incertidumbre natural y la epistémica. Aplican el planteamiento probabilista a los parámetros de rozamiento y cohesión y analizan la estabilidad de la presa en función del nivel de embalse, construyendo un conjunto de curvas de fragilidad que ayudan al ingeniero a entender y prevenir en la gestión de las presas. Es destacable mencionar como las curvas de fragilidad obtenidas confirman los resultados de investigaciones previas, y es que el fallo de la estructura se presenta repentinamente por lo que para conocer los márgenes de seguridad este enfoque será imprescindible.

En el estudio que planteamos en este artículo, se realiza una reevaluación determinista de los coeficientes de seguridad al deslizamiento de un conjunto de seis presas españolas, considerando las tensiones y aperturas de grieta obtenidas mediante modelos numéricos lineales y no lineales. Se comparan estos coeficientes con los originales, y se realizan adicionalmente sondeos mediante taladro para verificar el agrietamiento de la interfase presa-cimentación, junto con medidas de movimientos de la misma mediante extensómetros validando los resultados obtenidos.

\section{BASES DEL TRABAJO}

Aunque hay diversidad de estudios sobre la estabilidad al deslizamiento de presas, en aquellos en los que se ha tratado del parque de presas españolas de los años 50-60, no se analiza la diferencia de valores entre los que estimaron los proyectistas de la época y los que se obtendrían con las herramientas numéricas actuales. Tampoco es habitual encontrar referencias de inspecciones, ensayos y mediciones que puedan confirmar los resultados. Uno de los autores, por su desempeño laboral tiene acceso a la información técnica de un importante número de presas, y se han aprovechado actuaciones de mantenimiento (limpiezas de sistemas de drenajes, instalación de piezómetros o extensómetros) para obtener datos de investigación.

En España, las presas de gravedad, o contrafuertes, anteriores a 1970, constituyen en torno al $85 \%$ del patrimonio de presas de estas tipologías. La Instrucción (1) diferencia las solicitaciones en normales y accidentales; y aborda de manera independiente la disminución del ángulo de rozamiento y la cohesión. Nos ha parecido un obligado ejercicio de análisis como ingenieros a cargo de la seguridad y explotación de presas de esa época, el comparar los coeficientes a la estabilidad según estos criterios que significaron un punto de referencia y partida en la época, con los coeficientes obtenidos utilizando las herramientas numéricas y experimentales actuales. Estas herramientas se refieren tanto al cálculo como a los posibles sistemas de inspección y medición que sirvan para corroborar resultados. Con el objetivo de conocer con más exactitud el valor de los coeficientes y el margen para, llegado el caso, tomar medidas.

Los niveles de solicitaciones los definió el Reglamento de 1996 (2) para las presas públicas y las posteriores. En 2003 la Guía Técnica de Seguridad de Presas $N^{\circ} 2$ (3) define los períodos de retorno de las avenidas en función de la Categoría de las presas y su tipología acotando los diferentes niveles a considerar en los cálculos. Ahora, con la próxima aprobación de las Normas Técnicas, estos umbrales y solicitaciones se aplicarán a todas las grandes presas españolas y las pequeñas clasificadas como A o B en función de su riesgo potencial frente a la rotura, mediante la obligatoria Revisión de Seguridad periódica. Su entrada en vigor marcará un hito en España aplicando estos análisis y actualizaciones independientemente de la titularidad y edad de las presas. Esta razón respalda el análisis que se desarrolla en los siguientes apartados, con la intención de reflexionar sobre la necesidad y utilidad de los requerimientos que supondrá la aprobación de las Normas Técnicas, como un paso más en el control del riesgo de este tipo de infraestructuras. Para abordar esta cuestión, se han seleccionado seis presas en explotación construidas en la década 1955-1965:

- Prada en el río Jares. Presa de contrafuertes de $85 \mathrm{~m}$ de altura construida en el año 1958.

- San Sebastián en el río Bibey. Presa de contrafuertes de 60 m de altura construida en el año 1959.

- La Campañana en el río Balén-Sil. Presa de gravedad de 50 m de altura del año 1963.

- Montearenas en el río Boeza. Presa de gravedad de $37 \mathrm{~m}$ de altura y construida en 1965

- Las Ondinas en el río Sil. Presa de gravedad de $30 \mathrm{~m}$ de altura construida en el año 1963.

- Peñarrubia en el río Sil. Presa de gravedad de $36 \mathrm{~m}$ de altura finalizada en 1961.

Las presas de gravedad elegidas no son muy altas y se sitúan en ríos con caudales importantes (Noroeste), al tener el peso una mayor incidencia en la reducción del coeficiente de seguridad al deslizamiento. La razón es clara, las secciones más expuestas, o sea, con menor peso del hormigón y máxima altura de agua, son las del aliviadero, donde los huecos de las compuertas son proporcionalmente más importantes con respecto a la altura total de la presa, que en presas de mayor altura.

Sobre ellas, se realizan los cálculos de estabilidad según proyecto para embalse normal, para avenida con período de retorno de 500 años para conocer las exigencias de la Instrucción vigente hasta la fecha en estas presas, y para los criterios actuales con períodos de retorno de 1.000 y 10.000 años. Estos coeficientes se comparan con los calculados con modelos numéricos lineales de elementos finitos; y como comprobación de estos resultados se realizan modelos no lineales 
teniendo en cuenta la interfase y el comportamiento para el estado de carga más desfavorable.

Como comprobación experimental se ha investigado el cimiento de cada presa realizando diversos sondeos a rotación con corona de diamante y extracción de testigo. También se han instalado extensómetros para poder medir posibles movimientos relativos entre la presa-cimiento, complementando su auscultación. Aquellos que no rompieron por la interfase del contacto, se han ensayado a tracción. Los sondeos han permitido introducir una cámara de TV y poder detectar tanto grietas como flujos de agua, a cota máxima de embalse.

\section{ANÁLISIS NUMÉRICO}

Se ha calculado el coeficiente de seguridad al deslizamiento $\mathrm{K}$ en cada presa estudiada con los datos del estudio original y mediante modelos numéricos. Los niveles característicos, y por lo tanto estudiados, para todas las presas, han sido los siguientes:

- Nivel Máximo Normal de Embalse: nivel del embalse a la cota máxima de sus compuertas.

- Nivel Máximo según la Instrucción: es el alcanzado por la Onda de Avenida de 500 años, laminada.

- Nivel de la Avenida de Proyecto: es el de diseño de una presa según el Reglamento vigente. Corresponde a la Avenida de 1.000 años, laminada, de acuerdo con las Guías Técnicas del Comité Español de Grandes Presas.

- Nivel de la Avenida Extrema: es el de la máxima avenida que es capaz de soportar la presa sin que se produzca rotura. Corresponde como máximo a la de 10.000 años, laminada, según Guías Técnicas del Comité Español de Grandes Presas.

De acuerdo al artículo 39 de la Instrucción, se determinan los coeficientes $\mathrm{K}_{\mathrm{i}}$ de estabilidad al deslizamiento para las diferentes combinaciones de solicitaciones con la fórmula [1], siendo $\mathrm{N}$ la resultante de fuerzas normales, salvo la subpresión U, a la línea de deslizamiento, j el ángulo de rozamiento, c la cohesión, $\mathrm{S}$ la superficie de rotura sobre la que actúa la cohesión, T la resultante de fuerzas paralela a la superficie de rotura, F1 coeficiente de seguridad del rozamiento y F2 coeficiente de seguridad de la cohesión:

[1] $\quad K=\frac{\frac{(N-U)}{F 1} \operatorname{tg} \varphi+S \frac{c}{F 2}}{T}$
Denominamos $\mathrm{K}_{1}$ al coeficiente con arreglo a los criterios de diseño, en que se suponía resistencia a tracción en el contacto hormigón-roca suficiente para evitar el agrietamiento y distribución lineal de tensiones en la base. Este coeficiente abarca así los tres estados propuestos por la Instrucción (N.M.N. con y sin drenaje; Nivel Máximo) y los dos adicionales que propone el Reglamento (Avenida de Proyecto y Extrema).

Para cada estado de carga también se calcula el coeficiente $\mathrm{K}_{2}$ obtenido con un modelo de elementos finitos lineal en el que se calcula la distribución de tensiones verticales entre presa y terreno por peso propio y cargas hidrostáticas. Superponiendo la subpresión a las tensiones normales del modelo numérico se calcula el posible agrietamiento del contacto. El software utilizando ha sido el programa Abaqus 6.14 (26). Se muestra un ejemplo y la obtención del potencial agrietamiento para la avenida de proyecto en la presa de Peñarrubia (fig. 1).

En el caso de los modelos numéricos, con las longitudes de grieta calculadas se modifican los términos de subpresión y superficie de rotura. La subpresión se introduce bajo los supuestos de drenes eficaces e ineficaces. Los coeficientes parciales de disminución del ángulo de rozamiento y de la cohesión F1 y F2, aparecen en la tabla 1:

Tabla 1. Coeficientes de seguridad considerados

\begin{tabular}{|l|c|c|l|}
\hline HIPÓTESIS & $\mathbf{F}_{\mathbf{1}}$ & $\mathbf{F}_{\mathbf{2}}$ & ORIGEN \\
\hline Embalse Normal & $\mathbf{1 , 5}$ & 5 & Instrucción y Guías Técnicas \\
\hline Avenida 500 años & $\mathbf{1 , 2}$ & 4 & Instrucción \\
\hline Avenida 1.000 años & $\mathbf{1 , 2}$ & 4 & Guías Técnicas \\
\hline Avenida 10.000 años & $\mathbf{1 , 0}$ & 3 & Guías Técnicas \\
\hline
\end{tabular}

La superposición del estado tensional por peso propio y cargas hidrostáticas, con el valor de la subpresión en cada estado para determinar el potencial agrietamiento supone que el contacto hormigón-roca no tiene resistencia a tracción, como aconseja el estado del arte. No se ha tenido en cuenta en ninguno de los casos, a efectos comparativos el borrador actual de las Normas Técnicas donde no diferencia las reducciones a aplicar a los valores del ángulo de rozamiento y de la cohesión.

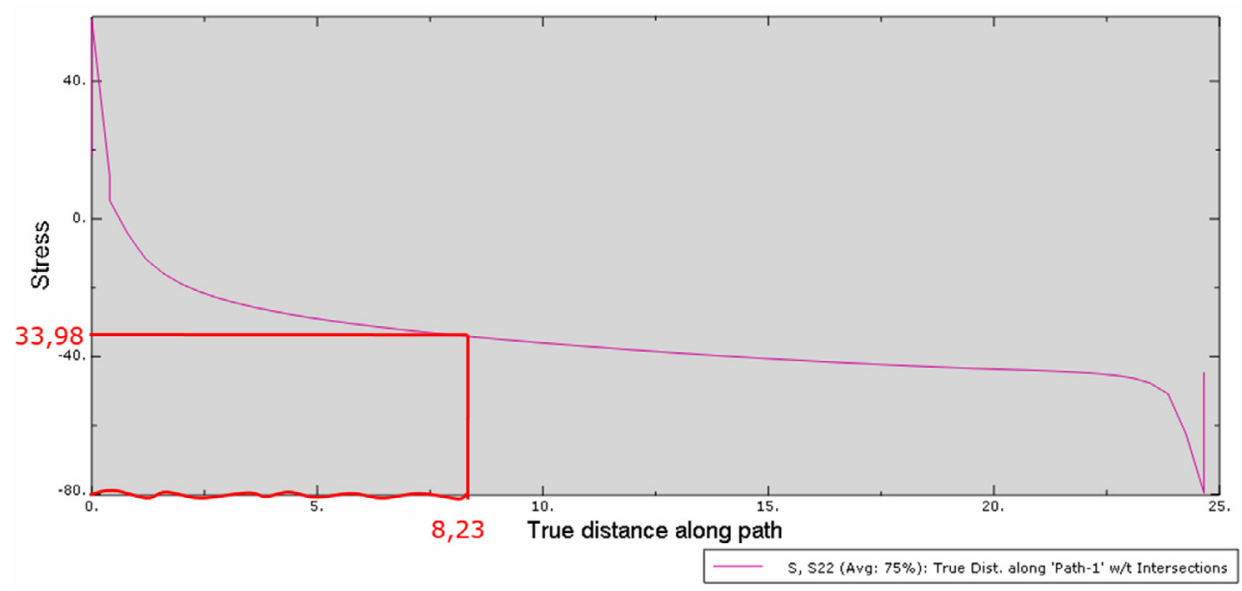


Las superficies de deslizamiento consideradas simplifican la interfase presa-roca como se muestra en los modelos de los próximos apartados. En las presas de gravedad la presa se supone en tensión plana y el terreno en deformación plana. En el caso de las dos presas de contrafuertes, se elaboró un modelo tridimensional de elementos finitos, del contrafuerte central y el terreno, calculando el agrietamiento al comparar, por un lado, la función de subpresión total sobre la base del contrafuerte, y por otro la resultante de las tensiones verticales para cada uno de los estados de carga (fig. 2), integrando las tensiones en el espesor del contrafuerte para simplificar el problema.
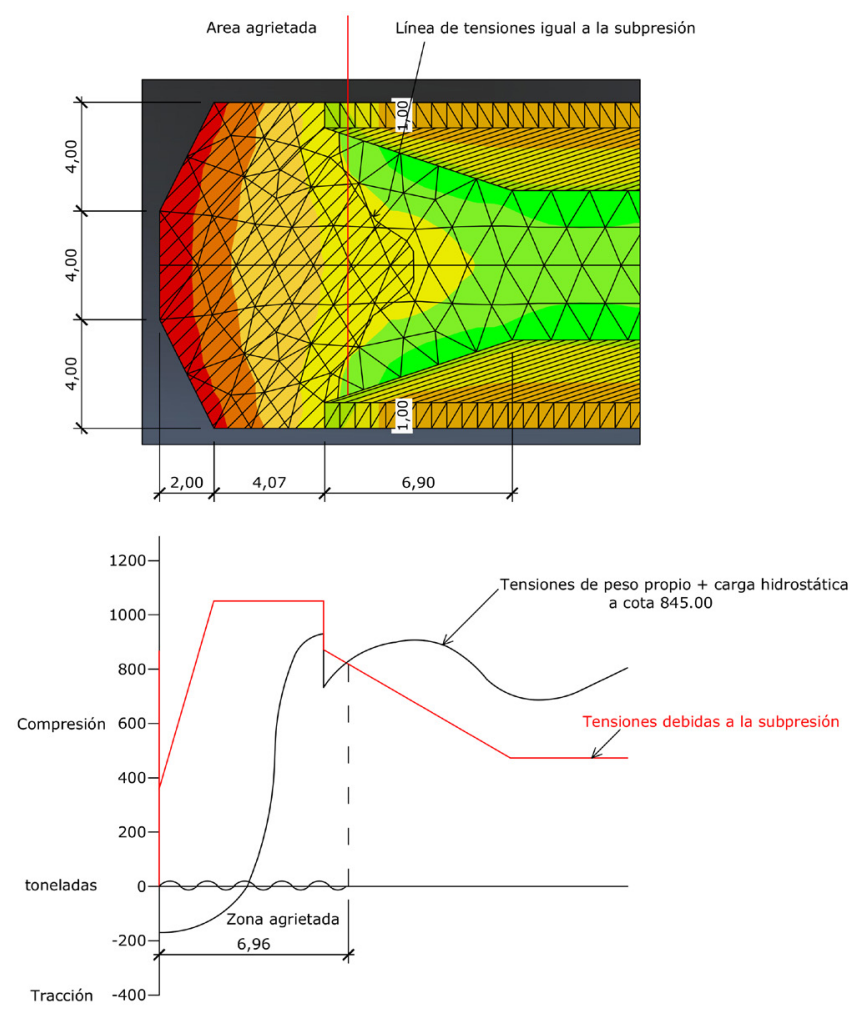

Figura 2. Tensión vertical y subpresión ( $\mathrm{t} / \mathrm{m}$ ) en la interfase presa roca del contrafuerte central de la presa de Prada en su sección media

En las presas de gravedad, tras calcular la longitud de agrietamiento potencial, se ha analizado la influencia del funcionamiento del conjunto, pantalla de impermeabilización-red de drenaje, considerando dos hipótesis (fig. 3):

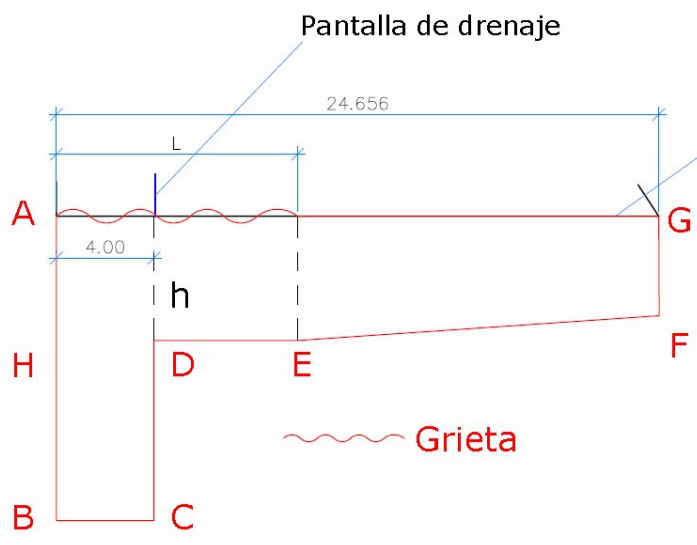

- En la primera hipótesis, se supone un caudal de filtración moderado en la grieta, unido a un drenaje eficaz, con una pérdida de carga de las filtraciones, en la línea de drenes de $1 / 3$ del total, lo habitual si el drenaje es efectivo. Se han calculado así los coeficientes llamados $\mathrm{K}_{2}$

- En la segunda hipótesis, se supone que el caudal de filtración supera largamente la capacidad del sistema de drenaje, convirtiendo al drenaje en ineficaz al permitir que aparezcan importantes subpresiones, lo que permite estudiar la influencia en la seguridad de una adecuada red de drenaje, tanto en diseño como en mantenimiento. Se han calculado así los coeficientes denominados $\mathrm{K}^{\prime \prime}{ }_{2}$.

En todos los casos se han considerado los valores de la resistencia al corte del contacto obtenidos de la documentación existente en el Archivo Técnico de la presa. No se han actualizado esos valores, ya que el objeto del trabajo ha sido comparar los coeficientes de seguridad del proyecto y los obtenidos con los modelos numéricos.

Con el fin de validar las aperturas de grieta obtenidas en los modelos lineales, se ha realizado un modelo considerando el contacto no lineal entre presa y terreno, mediante la definición de superficies de contacto cohesivas o no y con rozamiento, obteniendo resultados similares de longitudes de grieta. Adicionalmente, este modelo permite obtener los movimientos relativos en la interfaz y eliminar singularidades tensionales asociadas al modelo lineal. Los resultados obtenidos se presentan en el apartado siguiente, para la presa de Peñarrubia.

\subsection{Resumen de resultados}

Se presentan a continuación los resultados obtenidos en las presas consideradas. En los modelos numéricos las propiedades consideradas para el hormigón de las presas han sido: módulo de elasticidad $\mathrm{E}=3 \times 10^{6} \mathrm{t} / \mathrm{m}^{2}$, coeficiente de Poisson $v=0,2$ y peso específico $\rho=2,35 \mathrm{t} / \mathrm{m}^{3}$.

\section{Presa de Prada}

Fue una de las presas significativas de contrafuertes construidas en España en la primera mitad del siglo XX, sobre el río Jares, en la provincia de Ourense. El aliviadero es lateral a la presa. Su construcción data de 1958, y su proyecto de cuatro años antes. 


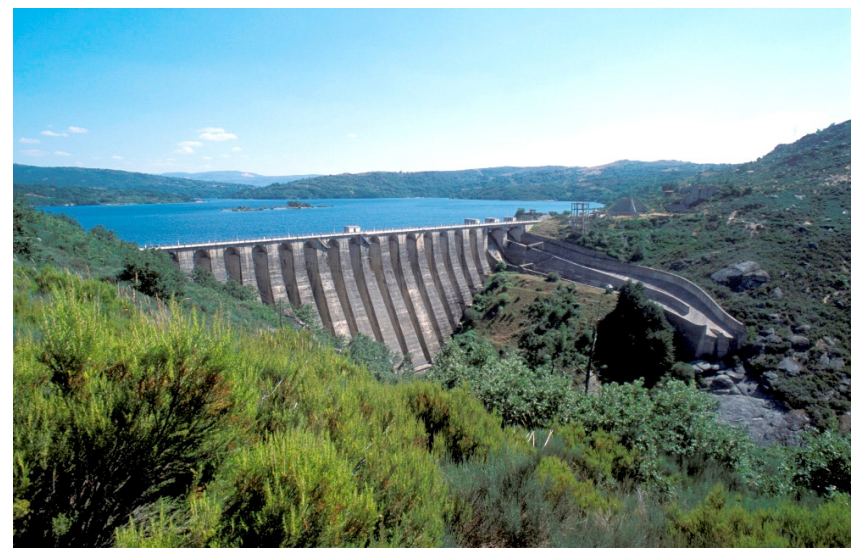

Figura 4. Vista de la presa de Prada

La cota de coronación es la 847 y la cota inferior de cimientos la 757 , tiene, por tanto, 90 metros de altura y crea un embalse de $121 \mathrm{hm}^{3}$. Los contrafuertes tienen taludes de 0,40 aguas arriba y de 0,55 aguas abajo, ancho de 12 metros y cabeza de diamante.

Se han utilizado los datos de la última actualización hidrológica del Archivo Técnico de la presa, de 2010. La avenida para un período de retorno de 500 años se cifra en $830 \mathrm{~m}^{3} / \mathrm{s}$ y la correspondiente a 10.000 años en $966 \mathrm{~m}^{3} / \mathrm{s}$. No hay apenas variación de niveles para las diferentes avenidas.

Para el cálculo de los coeficientes de seguridad se ha considerado un ángulo de rozamiento interno j en la interfaz de 45 ${ }^{o}$ y una cohesión c de $97,5 \mathrm{t} / \mathrm{m}^{2}$. Las propiedades del terreno deducidas a partir de los ensayos y geofísica de la época son $\mathrm{E}=2,5 \times 10^{6} \mathrm{t} / \mathrm{m}^{2}, \mathrm{y} v=0,2$. Los elementos finitos utilizados para modelar el contrafuerte y el terreno (fig. 5) son tetraedros lineales de 4 nudos con integración reducida $\left(\mathrm{C}_{3} \mathrm{D} 4\right)$.

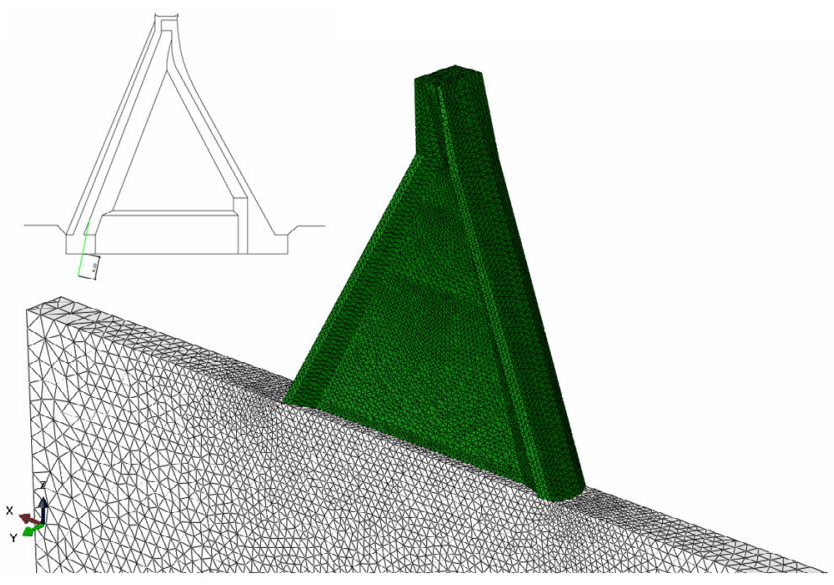

Figura 5. Sección tipo y modelo numérico de la presa de Prada

Los resultados obtenidos para los coeficientes de seguridad al deslizamiento se muestran en la tabla 2. Las diferencias en los coeficientes calculados con ambas metodologías se sitúan en torno al $\mathbf{1 0 \%}$.

La apertura máxima de grieta potencial que se ha obtenido es de 6,96 m (fig. 2). Para el N.M.N. los valores máximos de nivelación y colimación del modelo en coronación corresponden a $0,5 \mathrm{~mm}$ y $10 \mathrm{~mm}$ respectivamente. Los valores medidos por los equipos de auscultación han sido de $3 \mathrm{~mm}$ y 4,5 $\mathrm{mm}$ respectivamente. Las diferencias pueden deberse a los parámetros del modelo a falta de conocer mejores las características de los materiales, y a los propios sistemas de auscultación, actualmente en proceso de modernización.

Tabla 2. Coeficientes de seguridad Presa de Prada

\begin{tabular}{|l|c|c|c|}
\hline Situación & $\begin{array}{c}\text { Criterios de } \\
\text { diseño } \mathbf{K}_{\mathbf{1}}\end{array}$ & $\begin{array}{c}\text { Resultados } \\
\text { numéricos } \\
\mathbf{K}_{\mathbf{2}}\end{array}$ & $\mathbf{K}_{\mathbf{2}} / \mathbf{K}_{\mathbf{1}}$ \\
\hline N.M.N. & 1,30 & 1,18 & 0,91 \\
\hline Avenida 500 años & 1,63 & 1,47 & 0,90 \\
\hline Avenida 1.000 años & 1,63 & 1,47 & 0,90 \\
\hline Avenida 10.000 años & 1,97 & 1,80 & 0,91 \\
\hline
\end{tabular}

\section{Presa de San Sebastián}

Presa de contrafuertes construida sobre el río Bibey, con proyecto de 1959, por los profesores Vallarino y Alvárez. La cota de coronación es la 1.200,10 y la cota inferior de cimientos la 1.141,6o. El plano de cimentación es inclinado, formando un ángulo de $3,89^{\circ}$ con la horizontal. Su altura es menor que la de Prada, con 58,50 metros y la diferencia entre los taludes de los paramentos la hacen más esbelta. Su embalse almacena un total de $45 \mathrm{hm}^{3}$. Los datos hidrológicos fueron actualizados en 2011. La avenida para un período de retorno de 500 años es de $501 \mathrm{~m}^{3} / \mathrm{s}$ y la correspondiente a 10.000 años de $704 \mathrm{~m}^{3} / \mathrm{s}$.

Las propiedades utilizadas en el cálculo de los coeficientes de seguridad y en el modelo numérico (fig. 6) coinciden con las presentadas en la presa de Prada. Se procede como en el caso anterior obteniendo los resultados de la tabla 3 .

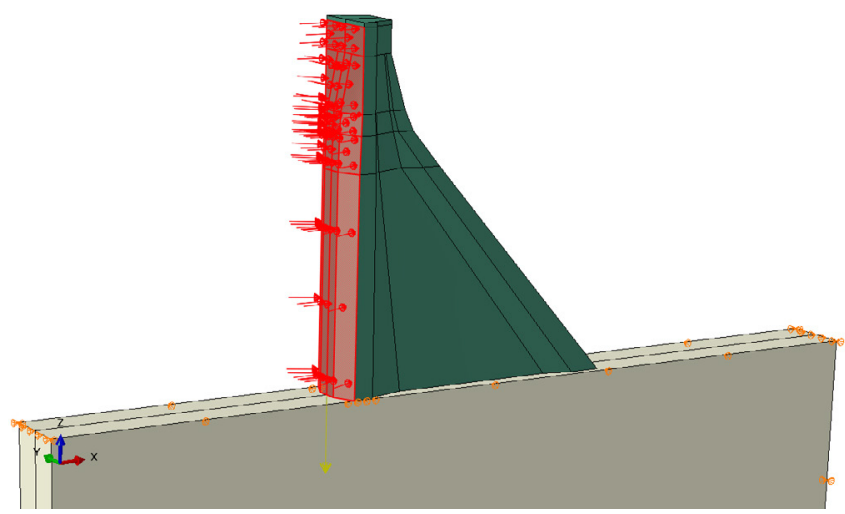

Figura 6. Geometría del modelo de contrafuerte, condiciones de contorno y carga hidrostática a cota $57,3 \mathrm{~m}$

Tabla 3. Coeficientes de seguridad Presa de San Sebastián

\begin{tabular}{|l|c|c|c|}
\hline Situación & $\begin{array}{c}\text { Criterios de } \\
\text { diseño } \mathbf{K}_{\mathbf{1}}\end{array}$ & $\begin{array}{c}\text { Resultados } \\
\text { numéricos } \\
\mathbf{K}_{\mathbf{2}}\end{array}$ & $\mathbf{K}_{\mathbf{2} / \mathbf{K}} \mathbf{K}_{\mathbf{1}}$ \\
\hline N.M.N. & 1,76 & 1,57 & 0,89 \\
\hline Avenida 500 años & 2,16 & 1,91 & 0,88 \\
\hline Avenida 1.000 años & 2,13 & 1,86 & 0,87 \\
\hline Avenida 10.000 años & 2,56 & 2,15 & 0,84 \\
\hline
\end{tabular}

La mayor diferencia en los coeficientes para la presa de San Sebastian se ha cifrado en un $\mathbf{1 6 \%}$ para solicitaciones extremas, siendo de un $\mathbf{1 1 \%}$ para las solicitaciones normales. Las aperturas 
máximas de grieta potencial que se han obtenido varían desde los 5,26 m (N.M.N.) hasta los 7,53 m (Avenida de 10.000 años).

Para el N.M.N. los valores máximos de nivelación y colimación del modelo en coronación corresponden a $2 \mathrm{~mm}$ y 3,5 mm respectivamente. Los valores medidos por los equipos de auscultación han sido de 2,20 mm y 5,5 $\mathrm{mm}$ respectivamente.

Es interesante resaltar como los autores en el proyecto cifran el valor de la subpresion en una distribución lineal a lo largo de la cabeza del contrafuerte, con un máximo aguas arriba de $\theta=0,75 \mathrm{H}$ pero sin considerar agrietamiento (fig. 7).
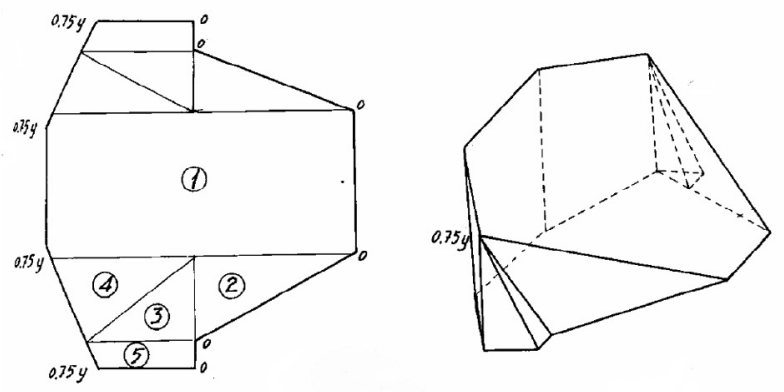

Figura 7. Presa de San Sebastian. Esquema de cálculo de subpresiones del proyecto original

\section{Presa de Ondinas}

Presa de gravedad construida sobre el Sil en el municipio de Palacios del Sil (León). Es un ejemplo de presa relativamente baja sobre un cauce con avenidas importantes y con compuertas de altura significativa (fig. 8). El talud aguas abajo de
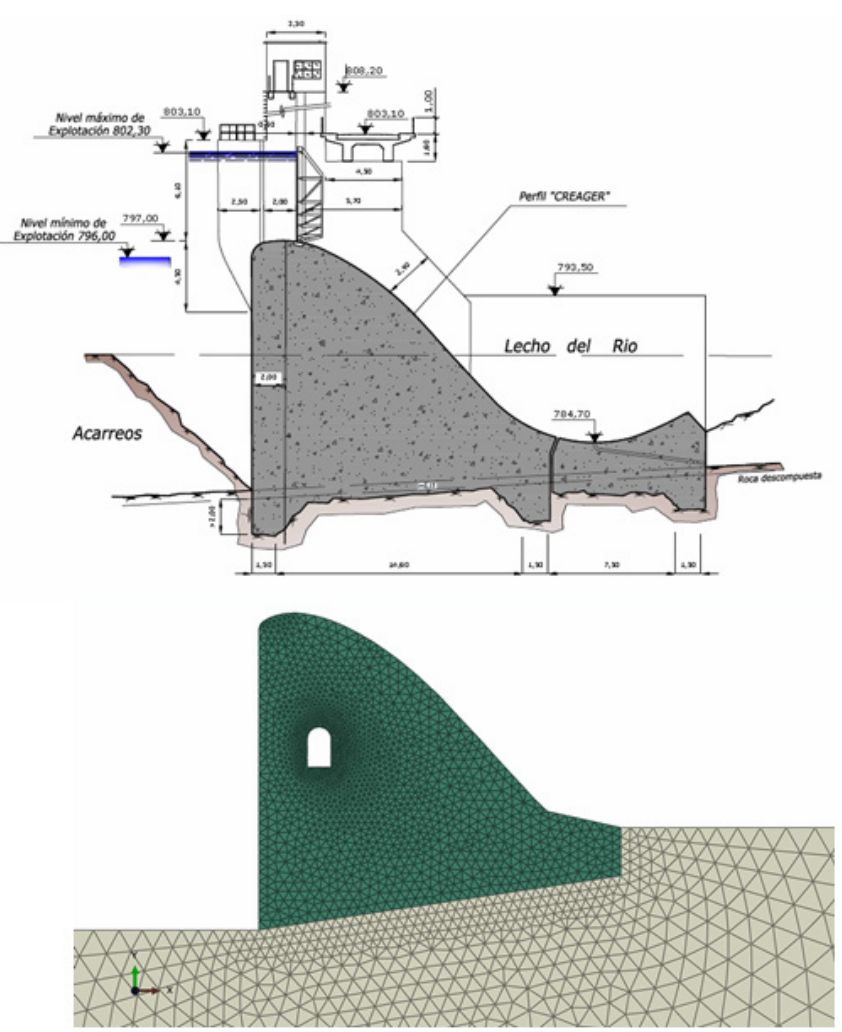

Figura 8. Sección tipo y modelo numérico de la presa de Ondinas
0,95:1,00 es muy superior a los usuales, el triángulo resistente con vértice a cota de coronación de presa, por encima de lo normal, y fuerte contrapendiente del contacto hormigón-roca, hacen de esta presa un caso singular en las de su época. Es la presa de mayor relación peso-empuje hidrostático de las presas estudiadas.

La cota de coronación es la 803,10, el Nivel Máximo Normal es la 802,65 y la cota inferior de cimientos es la 780,55. El plano de cimentación es inclinado con contrapendiente del $15 \%$. La altura máxima de la presa es de 22,55 metros. El volumen de embalse no llega al hectómetro, es una infraestructura de derivación.

El último estudio hidrológico de la cuenca es de 2012. La avenida para un período de retorno de 500 años se cifra en 1.148 $\mathrm{m}^{3} / \mathrm{s}$ y la correspondiente a 10.000 años en $1.569 \mathrm{~m}^{3} / \mathrm{s}$, superándose en un metro la coronación. Como particularidad, esta presa es la única de las cuatro analizadas que no dispone de red de drenaje.

$\mathrm{Al}$ existir un nivel de pizarra descompuesta paralelo a la cimentación de la presa, con litoclasado en esa dirección, se consideró en el Proyecto Base que la cohesión era nula, y el rozamiento, por el tableteado del plano de cimentación de $45^{\circ}$.

Los modelos se han realizado con elementos triangulares lineales. Los resultados obtenidos se muestran en la tabla 4.

Tabla 4. Coeficientes de seguridad Presa de Ondinas.

\begin{tabular}{|l|c|c|c|}
\hline \multicolumn{1}{|c|}{ Situación } & $\begin{array}{c}\text { Criterios de } \\
\text { diseño } \mathbf{K}_{\mathbf{1}}\end{array}$ & $\begin{array}{c}\text { Resultados } \\
\text { numéricos } \\
\mathbf{K}_{\mathbf{2}}\end{array}$ & $\mathbf{K}_{\mathbf{2} / \mathbf{K}_{\mathbf{1}}}$ \\
\hline N.M.N. & 1,13 & 1,10 & 0,97 \\
\hline Avenida 500 años & 1,40 & 1,36 & 0,97 \\
\hline Avenida 1.00o años & 1,34 & 1,26 & 0,94 \\
\hline Avenida 10.000 años & 1,46 & 1,27 & 0,87 \\
\hline
\end{tabular}

Las aperturas máximas de grieta potencial obtenidas varían desde los 0,78 m (N.M.N.) hasta los 1,87 m (Avenida de 10.000 años), con una longitud total de la base de 18,97 $\mathrm{m}$. Valores relativamente bajos comparados con el resto de presas. Para el N.M.N. los valores máximos verticales y horizontales de movimientos del modelo corresponden a 0,13 $\mathrm{mm}$ y $3,5 \mathrm{~mm}$ respectivamente, pero medidos en el propio aliviadero. Los valores medidos por los equipos de auscultación en coronación han sido de 1,50 mm de nivelación y 3,0 mm de colimación.

En el caso de la presa Las Ondinas, donde el diseño, 1963, correspondió a criterios muy conservadores, se obtienen unos resultados que sólo difieren en un $\mathbf{3 \%}$ para solicitaciones normales y un $\mathbf{1 3 \%}$ para extremas en los valores de los coeficientes de seguridad al deslizamiento frente a los criterios de diseño. Es conveniente señalar que, a pesar de los valores absolutos bajos de los coeficientes en los diferentes escenarios, estos siguen manteniendo su valor utilizando métodos numéricos y demuestran que el diseño no ha necesitado red de drenaje pues no es compatible según los resultados un agrietamiento que desestabilice el sistema presa-cimiento. A diferencia del resto de modelos bidimensionales calculados, no han aparecido tracciones en el tacón de aguas arriba de la presa para ninguno de los estados de carga. 


\section{Presa de Campañana}

Presa de gravedad construida en la cuenca del Sil que fue en su día un prototipo, creación del profesor Alfonso Álvarez, con aliviadero de labio fijo y de mucha mayor longitud que el cauce y que el cuenco amortiguador. El diseño vino condicionado por la geometría del macizo, constituido por pizarras que alternan con bancos delgados de cuarcitas [15]. Para compensar el diferente comportamiento de los suelos junto a la estratificación normal al cauce y el buzamiento de unos $80^{\circ}$ hacia aguas arriba, se buscó una solución tipo de gravedad con aumento de la seguridad al deslizamiento, repartiendo el talud total entre los dos paramentos (0,2 aguas arriba y o,6 aguas abajo) para contar con el empuje vertical estabilizador.

La cota de coronación es la 528,35, tiene una longitud de 175,00 m, el Nivel Máximo Normal es 525,80 y la cota inferior es la 482,35 . Su altura es de $46 \mathrm{~m}$ y forma un embalse de $14,74 \mathrm{hm}^{3}$.

Los datos hidrológicos fueron actualizados en 2012. La avenida para un período de retorno de 500 años se cifra en $75,47 \mathrm{~m}^{3} / \mathrm{s}$ y la correspondiente a 10.000 años en 80,92 $\mathrm{m}^{3} / \mathrm{s}$. En este caso tan sólo se superan 0,72 m el N.M.N. La finalidad del embalse, es alimentar una central y carece apenas de cuenca natural.

La presa se asienta sobre paquetes subverticales de pizarras muy duras y transversales al cauce. La línea de rotura más probable es una horizontal que pasa por el pie del talón por ser la cinemáticamente compatible más probable. Esta superficie irá rompiendo la cohesión de las discontinuidades existentes en el contacto hormigón-roca o la propia resistencia de la pizarra.

Se adoptan en este caso dos valores de cohesión en los cálculos $c_{1}=85 \mathrm{t} / \mathrm{m}^{2}$ para la rotura en el contacto hormigón-roca y $\mathrm{c}_{2}=126 \mathrm{t} / \mathrm{m}^{2}$ para rotura por la roca. El ángulo de rozamiento considerado es de $45^{\circ}$, y en el modelo numérico el terreno tiene las siguientes propiedades: $\mathrm{E}=1,5$ $\mathrm{x} 10^{6} \mathrm{t} / \mathrm{m}^{2}, \mathrm{y} v=0,25$.

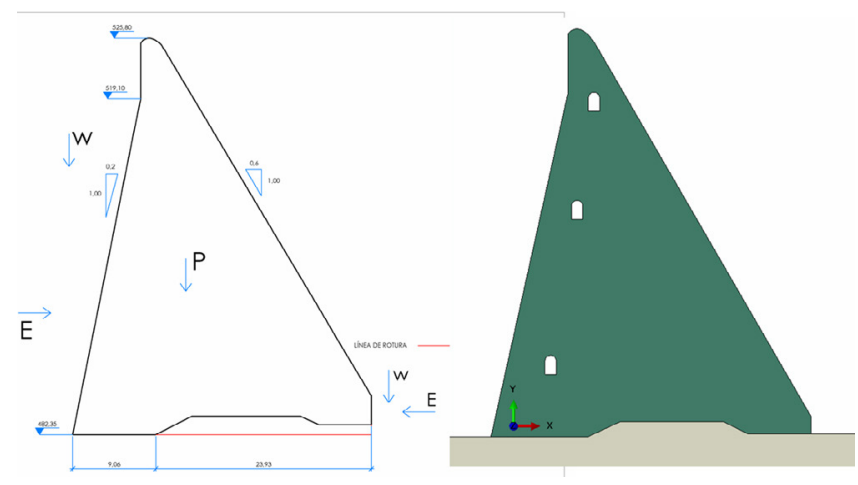

Figura 9. Sección tipo y modelo numérico de la presa de Campañana

Los resultados obtenidos si el sistema de drenaje es suficiente $\left(\mathrm{K}^{\prime}{ }_{2}\right)$ se muestran en la tabla 5 , mientras que en la tabla 6 se presentan los resultados $\left(\mathrm{K}^{\prime \prime}{ }_{2}\right)$ con un sistema de drenaje insuficiente que no es capaz de evacuar los caudales asociados al agrietamiento en un caso de apertura de grieta amplia.
Tabla 5. Coeficientes de seguridad Presa de Campañana. $\mathrm{K}_{2}{ }_{2}$

\begin{tabular}{|c|c|c|c|}
\hline Situación & $\begin{array}{c}\text { Criterios de } \\
\text { diseño } K_{1}\end{array}$ & $\begin{array}{c}\text { Resultados } \\
\text { numéricos } \\
\mathbf{K}_{2}^{\prime}\end{array}$ & $\mathbf{K}_{2 /}^{\prime} \mathbf{K}_{1}$ \\
\hline N.M.N. (drenaje eficaz) & 1,92 & 1,79 & 0,93 \\
\hline N.M.N. (drenaje ineficaz) & 2,07 & 1,88 & 0,91 \\
\hline Avenida 500 años & 2,33 & 2,13 & 0,91 \\
\hline Avenida 1.00o años & 2,33 & 2,13 & 0,91 \\
\hline Avenida 10.000 años & 2,93 & 2,66 & 0,91 \\
\hline
\end{tabular}

Tabla 6. Coeficientes de seguridad Presa de Campañana. $\mathrm{K}^{\prime \prime}{ }_{2}$

\begin{tabular}{|c|c|c|c|}
\hline Situación & $\begin{array}{c}\text { Criterios de } \\
\text { diseño } K_{1}\end{array}$ & $\begin{array}{c}\text { Resultados } \\
\text { num. K' }{ }_{2}^{\prime}\end{array}$ & $\begin{array}{c}\mathbf{K}^{\prime \prime}{ }^{2 /} \\
\mathbf{K}_{1}\end{array}$ \\
\hline N.M.N. (drenaje eficaz) & 1,92 & 1,50 & 0,78 \\
\hline N.M.N. (drenaje ineficaz) & 2,07 & 1,88 & 0,91 \\
\hline Avenida 500 años & 2,33 & 1,77 & 0,76 \\
\hline Avenida 1.00o años & 2,33 & 1,77 & 0,76 \\
\hline Avenida 10.000 años & 2,93 & 2,06 & 0,70 \\
\hline
\end{tabular}

Las aperturas máximas de grieta potencial obtenidas varían desde los 4,75 m (N.M.N.) hasta los 5,76 m (Avenida de 10.000 años), de una longitud total de la base de $33 \mathrm{~m}$. Valores superiores a los obtenidos en Ondinas, y donde ya se observa la necesidad del dimensionamiento de la red de drenaje y de su mantenimiento.

Para el N.M.N. los valores máximos verticales y horizontales de movimientos del modelo corresponden a 2,30 mm y 1,60 mm respectivamente, medidos en el aliviadero. Los valores medidos por los equipos de auscultación en coronación han sido de $2 \mathrm{~mm}$ de nivelación y de colimación.

En este caso se obtienen unos resultados en los valores de los coeficientes de seguridad que sólo difieren en un 7\% para solicitaciones normales y un $\mathbf{9 \%}$ para extremas. En cambio, si la apertura del agrietamiento supera la capacidad del sistema de drenaje, estos valores aumentan hasta diferenciar en un 22\% y un 30\% los coeficientes calculados con los modelos numéricos.

\section{Presa de Montearenas}

Presa de gravedad de planta ligeramente arqueada, construida sobre el río Boeza afluente del río Sil. La cota de coronación es la 552,00; el N.M.N. es la 549 y la cota inferior de cimientos la 515,25 . La base de la presa está en contrapendiente formando $3,95^{\circ}$ con la horizontal. Con una altura de $36,75 \mathrm{~m}$ sirve de trasvase de las aguas para derivarlas a su central. Su embalse es de $1,8 \mathrm{hm}^{3}$ y son frecuentes episodios de vertidos por compuertas pues se trata de un río caudaloso y poco regulado.

Los datos hidrológicos fueron actualizados en 2012. La avenida para un período de retorno de 500 años es de $1.476 \mathrm{~m}^{3} / \mathrm{s} \mathrm{y}$ la correspondiente a 10.000 años de $2.124 \mathrm{~m}^{3} / \mathrm{s}$. En este caso se supera en 2,1 m la cota de coronación. La presa (fig. 10) se sitúa sobre un granito sano, y la rotura se producirá a través de una superficie continua que irá rompiendo la cohesión de las discontinuidades existentes en el contacto hormigón-roca. Se considera una cohesión de $97,5 \mathrm{t} / \mathrm{m}^{2}$, un ángulo de rozamiento interno de $45^{\circ}$, y en el modelo numérico el terreno tiene las siguientes propiedades: $\mathrm{E}=2,8 \times 10^{6} \mathrm{t} / \mathrm{m}^{2}, \mathrm{y} v=0,25$. 


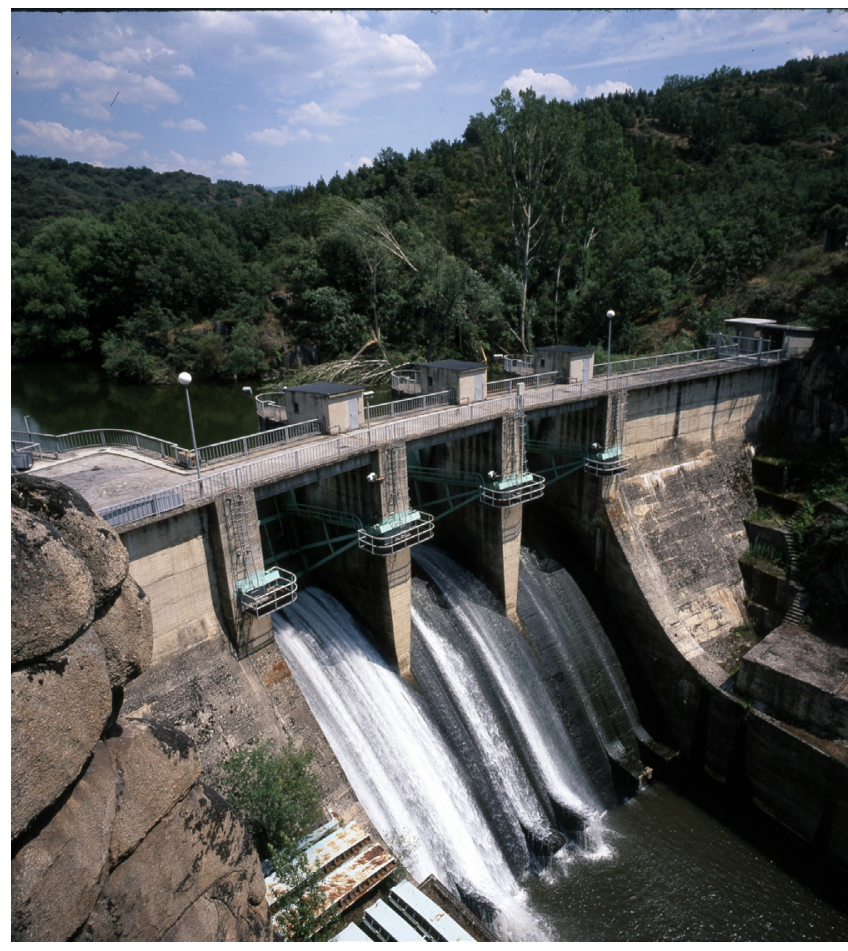

Figura 10. Vista de la presa de Montearenas

Los resultados suponiendo que el sistema de drenaje es suficiente para evacuar las posibles subpresiones aparecen en la tabla 7 , mientras que en el caso con drenaje insuficiente se presentan en la tabla 8.

Tabla 7. Coeficientes de seguridad Presa de Montearenas. $\mathrm{K}^{\prime}{ }_{2}$

\begin{tabular}{|c|c|c|c|}
\hline Situación & $\begin{array}{c}\text { Criterios de } \\
\text { diseño } K_{1}\end{array}$ & $\begin{array}{c}\text { Resultados } \\
\text { num. } \mathrm{K}^{\prime}{ }_{2}\end{array}$ & $\begin{array}{c}\mathbf{K}^{\prime}{ }_{2 /} \\
\mathbf{K}_{1}\end{array}$ \\
\hline N.M.N. (drenaje eficaz) & 1,94 & 1,56 & 0,80 \\
\hline N.M.N.(drenajeineficaz) & 2,03 & 1,46 & 0,72 \\
\hline Avenida 500 años & 2,27 & 1,76 & 0,78 \\
\hline Avenida 1.000 años & 2,20 & 1,69 & 0,77 \\
\hline Avenida 10.000 años & 2,54 & 1,87 & 0,74 \\
\hline
\end{tabular}

Tabla 8. Coeficientes de seguridad Presa de Montearenas.K' ${ }_{2}$

\begin{tabular}{|l|c|c|c|}
\hline Situación & $\begin{array}{c}\text { Criterios de } \\
\text { diseño K }\end{array}$ & $\begin{array}{c}\text { Resultados } \\
\text { num. } \mathbf{K}^{\prime}{ }_{\mathbf{2}}\end{array}$ & $\begin{array}{c}\mathbf{K}^{\prime}{ }^{\prime}{ }^{2 /} \\
\mathbf{K}_{\mathbf{1}}\end{array}$ \\
\hline N.M.N. (drenaje eficaz) & 1,94 & 1,16 & 0,60 \\
\hline N.M.N.(drenaje ineficaz) & 2,03 & 1,46 & 0,72 \\
\hline Avenida 500 años & 2,27 & 1,25 & 0,55 \\
\hline Avenida 1.ooo años & 2,20 & 1,18 & 0,54 \\
\hline Avenida 10.00o años & 2,54 & 1,28 & 0,50 \\
\hline
\end{tabular}

En este caso las aperturas máximas de grieta potencial son muy superiores tanto en valor absoluto como en lo relativo a su longitud total de contacto. Varían desde los 7,53 m (N.M.N.) hasta los 8,87 m (Avenida de 10.000 años), con una longitud total de la base de 24,12 m. Para el N.M.N. los valores máximos de movimientos verticales y horizontales del modelo corresponden a $0,20 \mathrm{~mm}$ y $1,84 \mathrm{~mm}$ respectivamente, medidos en el aliviadero. Los valores máximos medidos por los equipos de auscultación en coronación han sido de $1 \mathrm{~mm}$ de nivelación y $2 \mathrm{~mm}$ de colimación.

\section{Presa de Peñarrubia}

Presa de gravedad en el río Sil (fig. 11) que alimenta la central hidroeléctrica de Quereño a través de un canal de $140 \mathrm{~m}^{3} / \mathrm{s}$. La cota de coronación es la 397,42; su N.M.N. es la 394,42 y la cota inferior del cimiento es la 363,60. Su altura de 33,82 $\mathrm{m}$ y cierra un embalse de $12,6 \mathrm{hm}^{3}$.

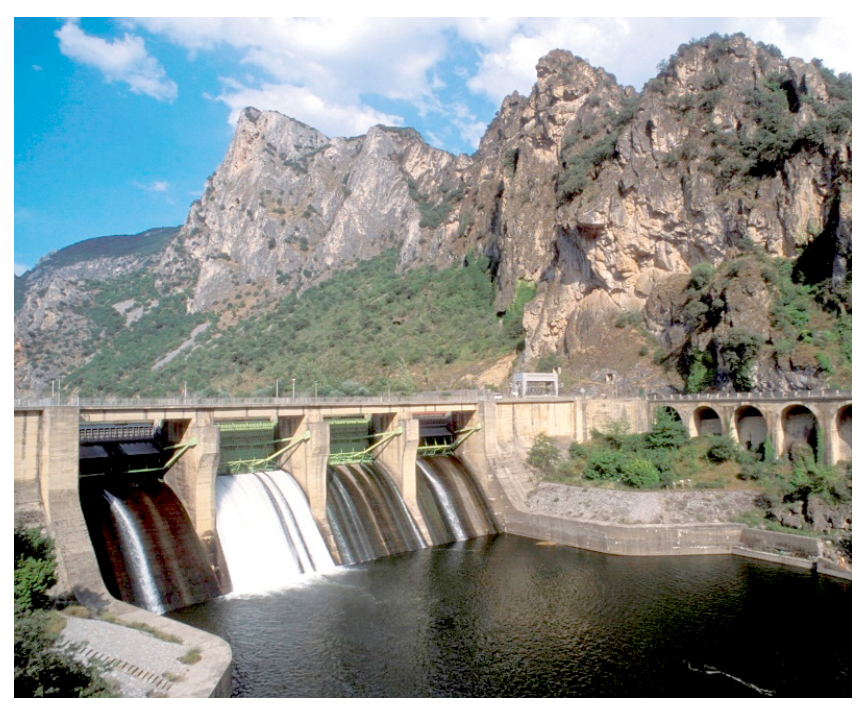

Figura 11. Vista de la presa de Peñarrubia

Su hidrología se actualizó en 2014. La avenida para un periodo de retorno de 500 años es de $4.178 \mathrm{~m}^{3} / \mathrm{s}$ y la correspondiente a 10.000 años de $5.760 \mathrm{~m}^{3} / \mathrm{s}$. En esta situación la lámina de agua supera la coronación en 2,24 m. En la cola de embalse confluyen los ríos Burbia y Cúa con importantes caudales y sin regular, lo que hace que los vertidos en Peñarrubia sean frecuentes.

La cerrada está constituida por pizarras sanas, pero diaclasadas. No existen litoclasas horizontales y con esta disposición de la geometría estructural del macizo la línea pésima de rotura solo puede ser el contacto hormigón-roca. Al ser menor el esfuerzo cortante del hormigón que el de la pizarra sana, se adopta el valor del primero. La resistencia debida a la cohesión según los datos del Archivo Técnico es $97,5 \mathrm{t} / \mathrm{m}^{2}$. Respecto al ángulo de rozamiento interno se considera un valor de $45^{\circ}$, y en el modelo numérico se consideran las siguientes propiedades del terreno: $\mathrm{E}=2 \times 10^{6}$ $\mathrm{t} / \mathrm{m}^{2}, \mathrm{y} v=0,2$.

Los resultados con sistema de drenaje suficiente para la apertura de grieta aparecen en la tabla 9, y los correspondientes a un drenaje insuficiente en la tabla 10.

Tabla 9. Coeficientes de seguridad Presa de Peñarrubia. $\mathrm{K}^{\prime}{ }_{2}$

\begin{tabular}{|l|c|c|c|}
\hline Situación & $\begin{array}{c}\text { Criterios de } \\
\text { diseño } \mathbf{K}_{\mathbf{1}}\end{array}$ & $\begin{array}{c}\text { Resultados } \\
\text { num. } \mathbf{K}^{\prime}{ }_{\mathbf{2}}\end{array}$ & $\begin{array}{c}\mathbf{K}^{\prime}{ }_{\mathbf{~} /} \\
\mathbf{K}_{\mathbf{1}}\end{array}$ \\
\hline N.M.N. (drenaje eficaz) & 2,03 & $\mathbf{1 , 8 3}$ & 0,90 \\
\hline N.M.N.(drenaje ineficaz) & 2,11 & 1,86 & 0,88 \\
\hline Avenida 500 años & 2,36 & 1,90 & 0,81 \\
\hline Avenida 1.000 años & 2,30 & 1,81 & 0,79 \\
\hline Avenida 10.000 años & 2,65 & 1,94 & 0,73 \\
\hline
\end{tabular}


Tabla 10. Coeficientes de seguridad Presa de Peñarrubia. $\mathrm{K}^{\prime \prime}{ }_{2}$

\begin{tabular}{|l|c|c|c|}
\hline Situación & $\begin{array}{c}\text { Criterios de } \\
\text { diseño } \mathbf{K}_{\mathbf{1}}\end{array}$ & $\begin{array}{c}\text { Resultados } \\
\text { num. } \mathbf{K}^{\prime}{ }_{\mathbf{2}}\end{array}$ & $\begin{array}{c}\mathbf{K}^{\prime \prime}{ }^{2 /} \\
\mathbf{K}_{\mathbf{1}}\end{array}$ \\
\hline N.M.N. (drenaje eficaz) & 2,03 & 1,49 & 0,73 \\
\hline N.M.N. (drenaje ineficaz) & 2,11 & 1,86 & 0,88 \\
\hline Avenida 500 años & 2,36 & 1,39 & 0,59 \\
\hline Avenida 1.o0o años & 2,30 & 1,30 & 0,57 \\
\hline Avenida 10.00o años & 2,65 & 1,31 & 0,49 \\
\hline
\end{tabular}

Es este el caso en el que las aperturas máximas de grieta potencial sufren la mayor variación respecto al empuje de todas las presas estudiadas, variando desde los 4,16 m (N.M.N.) hasta los 9,84 m (Avenida de 10.000 años), de una longitud total de la base de $24,65 \mathrm{~m}$.

La presa dispone de lecturas indirectas mediante manómetros en 13 puntos. Los valores hacían sospechar unas presiones más elevadas de lo habitual, por lo que se han instalado 8 piezómetros adicionales de cuerda vibrante aguas abajo de la pantalla para poder conocer de manera directa el efecto de la subpresión. Actualmente a cota N.M.N. la carga supera el $40 \%$ de carga de manera generalizada por lo que está previsto intervenir en la red de drenaje.

Los resultados obtenidos en la comparativa de coeficientes de seguridad difieren en un 10\% para solicitaciones normales y un 27\% para extremas. Pero si el sistema de drenaje no fuera capaz de evacuar el caudal, entonces estos coeficientes disminuyen un $\mathbf{2 7 \%}$ para solicitaciones normales y un $\mathbf{5 1 \%}$ para extremas.

Aunque los valores absolutos de los coeficientes de seguridad son suficientes, no impide que se deba ahondar en la investi- gación, por un lado, experimentalmente tratando de encontrar datos y pruebas que nos ayuden a definir parámetros más aproximados a la realidad, y por el otro lado numéricamente, analizando que niveles provocarían el agotamiento del sistema.

A la vista de los resultados y de la variación en la estabilidad al deslizamiento, se decidió mejorar el modelo numérico desarrollando un modelo no lineal para analizar la estabilidad al deslizamiento teniendo en cuenta la subpresión con la avenida entrante extrema. Para ello la interfase presa terreno se hace discontinua, y entre las dos superficies se define una interacción de contacto con propiedades de contacto rígido en dirección normal, es decir, penetraciones nulas y separación libre, y una ley tangencial de fricción elastoplastica aplicada mediante penalización. Adicionalmente se puede definir una cohesión en dirección normal que limite la separación libre.

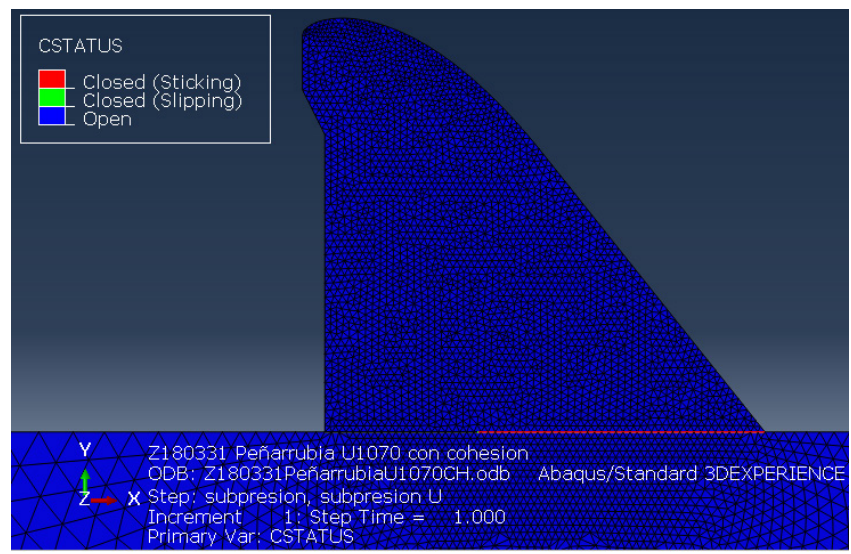

Figura 13. Estado del contacto en la interfaz con 10,70 m de grieta

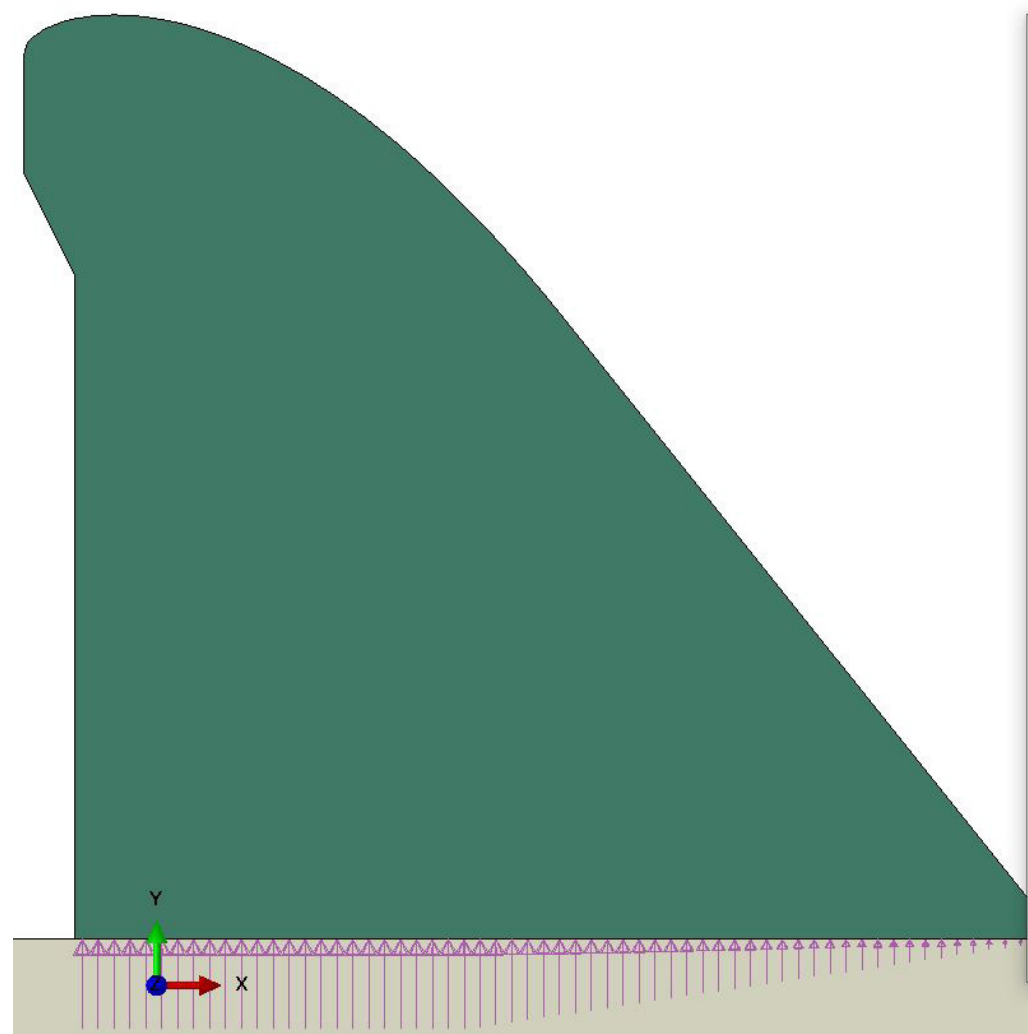

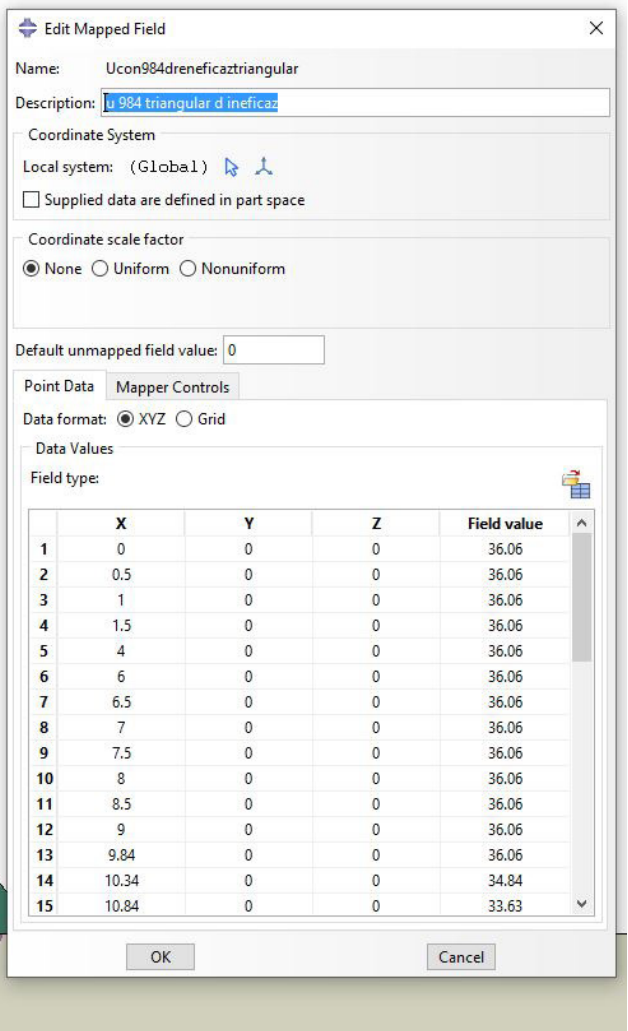


Para considerar el efecto de la subpresión en la grieta (fig. 12) se aplica un proceso iterativo en un bucle externo al de las iteraciones de contacto, en el que en función de la apertura de grieta resultante en cada paso se modifica la subpresión aplicada a lo largo de la grieta hasta que la longitud de esta se estabiliza. El análisis converge en un número de iteraciones razonable.

Considerando un coeficiente de rozamiento de 1 , avenida extrema y cohesión, la longitud de grieta converge a los 10,70 m superior a los $9.84 \mathrm{~m}$ del modelo lineal sin cohesión.

\section{TRABAJOS DE CAMPO}

Como complemento a los modelos se extrajeron 14 testigos de las presas de Campañana, Montearenas, Peñarrubia, Prada y San Sebastián y se instrumentaron con extensómetros de tres varillas por sondeo. Todos cortaban el contacto a pocos metros del paramento aguas arriba y profundizaban una media de 8 metros por debajo del contacto. La totalidad de los sondeos se realizaron con embalse a Nivel Máximo Normal, no puediendo realizar estos trabajos en situación de avenidas por seguridad.

Los taladros se realizaron a rotación con corona de diamante y recuperación de testigo de $\mathrm{f} 100 \mathrm{~mm}$, inspeccionando cada perforación con cámara de TV, caracterizándose con precisión la posición de la grieta en el contacto en los casos en los que se corroboró su existencia (fig. 14). De los 14 sondeos realizados, se comprobó, mediante la cámara, que, en 10, aparecía la grieta. En las 5 presas ha aparecido alguna zona del contacto despegada.

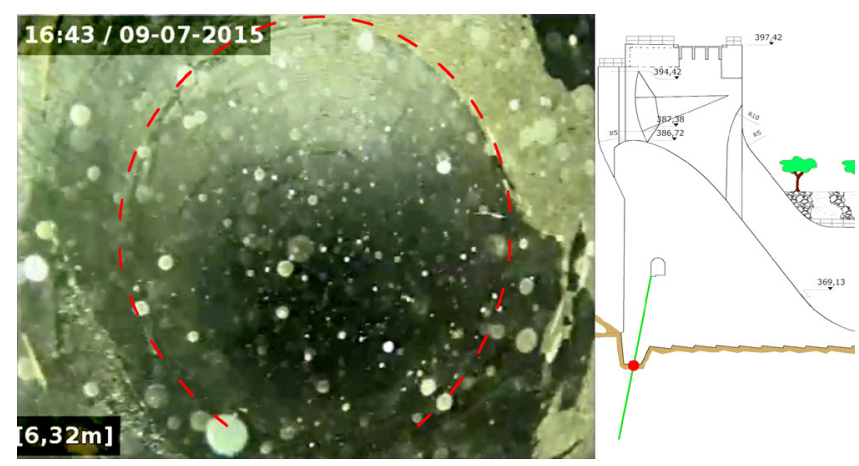

Figura 14. Presa de Peñarrubia, inspección con cámara. Grieta contacto roca-hormigón

En los casos de testigo sin grieta, se decidió ensayar a tracción las probetas para obtener el valor de rotura de la interfase, hormigón-roca. Las 4 perforaciones donde apareció la interfase unida, corresponden a 3 de las presas, sin embargo, el análisis del testigo, reflejaba que éste había roto por una litoclasa cercana al contacto. Esto demuestra que, la grieta se ha producido, pero no por el contacto, sino por una zona cercana, más débil de la roca, una litoclasa, de resistencia nula a tracción. Los resultados del ensayo se recogen en la tabla 11.

Tabla 11. Resistencias experimentales a tracción $\left(\mathrm{kg} / \mathrm{cm}^{2}\right)$

\begin{tabular}{|l|c|}
\hline Presa & Resistencia a tracción \\
\hline San Sebastián & 2,7 \\
\hline San Sebastián & 3,9 \\
\hline Montearenas & $6, \underline{2}$ \\
\hline La Campañana & 0,3 \\
\hline
\end{tabular}

Se produce una importante dispersión en los resultados, y destaca que las resistencias obtenidas son muy inferiores a las esperadas, sobre los $20 \mathrm{~kg} / \mathrm{cm}^{2}$. Atendiendo a los estudios norteamericanos EPRI, parece que la tecnología en España, al menos en los años 6o, de vibrado y compactado del hormigón, podría ser inferior a la de los EEUU.

De los 14 extensómetros instrumentados, se toman lecturas con frecuencia mensual desde su instalación analizándose los datos, aunque el volumen de datos no es aún amplio. El extensómetro que muestra una mayor amplitud de grieta es uno de los tres instalados en la presa de San Sebastían (fig. 15), con un valor máximo en el último ejercicio de $1,65 \mathrm{~mm}$ con variaciones de embalse de 21,45 metros y una relación directa con la carga.

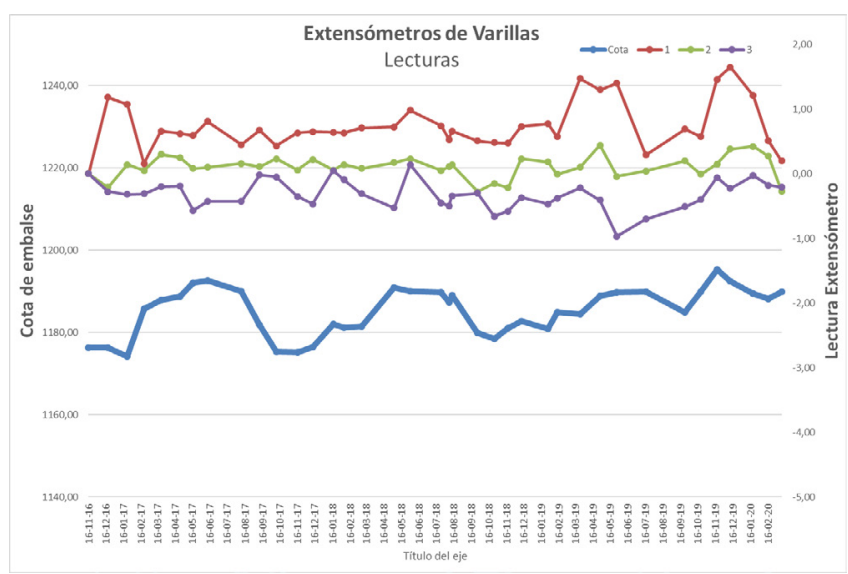

Figura 15. Lecturas extensómetros de varillas presa de San Sebastían

En las demás presas, salvo excepciones, se detectan comportamientos similares en tendencia, aunque con amplitudes menores. En el caso de Peñarrubia (fig. 16) donde la grieta potencial es mayor, dos de los cuatro extensómetros presentan una relación directa con la carga, con amplitudes de o,68mm para tan sólo una variación de cota de 3,66 m.

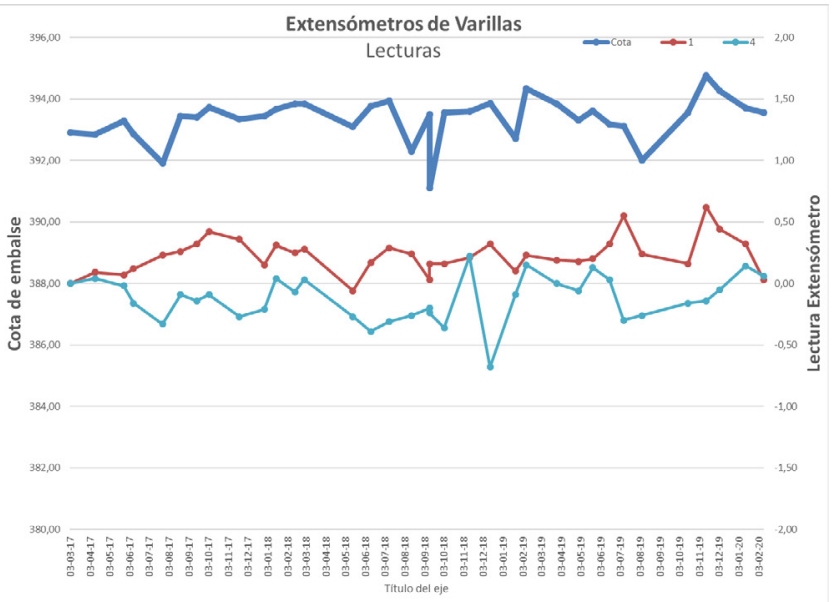

Figura 16. Lecturas extensómetros de varillas presa de Peñarrubia

\section{CONCLUSIONES Y RECOMENDACIONES}

\subsection{Conclusiones}

En base a los análisis realizados, se proponen las siguientes conclusiones: 
- Que las presas diseñadas con criterios clásicos, en nuestro caso, en los años 50-60, antes de que aparecieran métodos y herramientas de cálculo potentes, pueden tener importantes déficits de seguridad e incertidumbre al deslizamiento, máxime si la resistencia a tracción de la interfase es tan reducida como se ha comprobado. Creemos que es necesario analizar la estabilidad de todas nuestras grandes presas y las pequeñas que clasificadas como A o B en función de su riesgo potencial, como prescriben las Normas Técnicas actualizando los cálculos como aconseja el Estado del Arte.

- Se ha comprobado experimentalmente la existencia de agrietamiento en los casos en los que las resistencias del contacto o superficies del terreno cinemáticamente compatibles han sido menores que las tensiones. En este estudio, 10 de 14 inspecciones lo confirman como lo demuestran los testigos y los extensómetros.

- Que, en la mayoría de los casos, estas grietas son sensibles a los estados de carga y que pueden, mediante una adecuada instrumentación, contribuir al sistema de ausculatción de la presa y a mejorar la comprensión del comportamiento del conjunto.

- Se ha comprobado, en base a la extracción de testigos, que los parámetros de caracterización de los materiales en los cálculos de diseño pueden diferir de los reales.

\subsection{Recomendaciones}

- Priorizar las revisiones de seguridad en aquellas presas más antiguas y poniendo especial atención en las pequeñas y medianas emplazadas en ríos caudalosos.

- En las presas donde se prevea un posible agrietamiento en el tacón de aguas arriba, instrumentar los bloques con mayor afectación mediante extensómetros.

- En los casos donde se detecten movimientos, caracterizar con más detalle los bloques afectados, el contacto y el te- rreno para ajustar los resultados de los modelos numéricos de simulación de la interfase. Así se podrán ajustar con mayor precisión los valores de los coeficientes, y el nivel de seguridad de la presa.

- Sería aconsejable complementar los estudios determinísticos con estudios probabilistas. El cálculo de curvas de fragilidad para el modo de fallo al deslizamiento sería una herramienta fundamental para que el Ingeniero puede conocer el margen de seguridad de la infraestructura de la que es responsable.

- Es conveniente analizar la posibilidad de apertura repentina de grieta por un estado de carga excepcional, en base a los resultados debería analizarse si el sistema de drenaje es el adecuado. Para ello se requiere la comprobación de un correcto dimensionamiento de su capacidad de evacuación y un eficaz mantenimiento.

- Se habla de situaciones accidentales pero llegado el caso y en determinados bloques, quizá una ampliación de perforaciones en número y diámetro hasta el contacto de la presa y el terreno sirvieran como medida preventiva ante un fallo de la estructura por deslizamiento.

Finalmente concluir que, en todos los casos evaluados con el análisis numérico, los valores de los coeficientes al deslizamiento han sido superiores a la unidad para los diferentes estados de carga y podemos afirmar que las presas estudiadas siguen siendo estables. La aplicación de métodos actuales de cálculo y su complemento con ensayos e inspecciones nos ha permitido conocer mejor la certidumbre de los coeficientes de seguridad y complementar el estado real de la infraestructura. Podemos así decir que las revisiones de seguridad serán la herramienta esencial para que el Director de la presa pueda gestionar todas las labores de explotación, conservación y vigilancia con suficiente información, y criterios.

\section{REFERENCIAS / REFERENCES}

(1) Orden de 31 de marzo de 1967 por la que se aprueba la «Instrucción para proyecto, construcción y explotación de grandes presas». Boletín Oficial del Estado, núm. 257, de 27 de octubre de 1967, páginas 14716 a 14738. Recuperado de https://www.boe.es/boe/dias/1967/10/27/pdfs/A14716-14738.pdf

(2) Orden de 12 de marzo de 1996 por la que se aprueba el Reglamento Técnico sobre Seguridad de Presas y Embalses. Boletín Oficial del Estado, núm. 78, de 30 de marzo de 1996, páginas 12244 a 12254. Recuperado de https://www.boe.es/ boe/dias/1996/03/30/pdfs/A12244-12254.pdf

(3) Comité Nacional Español de Grandes Presas. (2003). Guía técnica de seguridad de presas número 2. Criterios para proyectos de presas y obras anejas. (Ed. rev.). Madrid, España: Comité Nacional Español de Grandes Presas.

(4) Real Decreto 9/2008, de 11 de enero, por el que se modifica el Reglamento del Dominio Público Hidráulico, aprobado por el Real Decreto 849/1986, de 11 de abril. Boletín Oficial del Estado, núm. 14, de 16 de enero de 2008, páginas 3141 a 3149. Recuperado de https://www.boe.es/buscar/doc.php?id=BOE-A-2008-755

(5) Proyecto de Real Decreto XXX/2019, por el que se aprueban las Normas Técnicas de Seguridad para las presas y sus embalses, Borrador sometido a información pública el 5 de septiembre de 2019 en la web del Ministerio. https://www.miteco.gob.es/es/agua/participacion-publica/PP-Proyecto-RD-Normas-Tecnicas-Seguridad-Presas-septbre-2019.aspx

(6) ICOLD. International Commission on Large Dams. (1995). Dam Failures - Statistical Analysis. Bulletin 99.

(7) Lévy, M. (1895, 5 de agosto; 1896, 2 de mayo; 1899, 4 de julio). Comunicaciones a la Academia de Ciencias de Francia.

(8) Bureau of Reclamation. United States Department of the Interior (1960). Design of small dams. USA: A Water Resources Technical Publication.

(9) Bureau of Reclamation. United States Department of the Interior (1976). Design of Gravity dams. Denver, Colorado: A Water Resources Technical Publication.

(10) Gómez Navarro, J.L. y Aracil Segura, J.J. (1958). Saltos de agua y presas de embalse. Madrid: Tipografía Artística.

(11) Zienkiewicz, O.C. (1971). The finite element method in engineering science. London: McGraw-Hill.

(12) Vallarino, E. (1995). Tratado básico de presas. Madrid: Paraninfo.

(13) Granados, A. (1995). Problemas de obras hidráulicas (pp. 149-217). Madrid: Colegio de Ingenieros de Caminos, Canales y Puertos.

(14) Linsbauer, H., \& Bhattacharjee, S. (1999). Dam safety assessment due to uplift pressure action in a dam-foundation interface crack. 5 Th Benchmark Workshop On Numerical Analysis Of Dams. Denver. 
(15) Ruggeri, G. (2004). Working Group on uplift pressures under concrete dams - Final Report. ICOLD European Club.

(16) Ruggeri, G. (2004). Working Group on sliding safety of existing gravity dams - Final Report. ICOLD European Club.

(17) Cai, Q. (2007). Finite element modelling of cracking in concrete gravity dams (Tesis doctoral). University of Pretoria.

(18) Barpi, F., \& Valente, S. (2010). The cohesive frictional crack model applied to the analysis of the dam-foundation joint. Engineering Fracture Mechanics, 77(11), 2182-2191. doi: 10.1016/j.engfracmech.2010.02.030

(19) Moftakhar, M., \& Ghafouri, H. (2011). Comparison of Stability Criteria for Concrete Dams in Different Approximate Methods Based on Finite Element Analysis. Procedia Engineering, 14, 1672-1680. doi: 10.1016/j.proeng.2011.07.210

(20) McKay, M., \& López, F. (2013). Practical methodology for inclusion of uplift and pore pressures in analysis of concrete dams. Multiple Use Of Dams And Reservoirs: Needs, Benefits And Risks.IPENZ Proceedings Of Technical Groups 39 (LD).

(21) Krounis, A., Johansson, F., \& Larsson, S. (2015). Effects of spatial variation in cohesion over the concrete-rock interface on dam sliding stability. Journal of Rock Mechanics and Geotechnical Engineering, 7(6), 659-667. doi: 10.1016/j. jrmge.2015.08.005.

(22) Altarejos-García, L., Escuder-Bueno, I., Serrano-Lombillo, A., \& de Membrillera-Ortuño, M. (2012). Methodology for estimating the probability of failure by sliding in concrete gravity dams in the context of risk analysis. Structural Safety, 36-37, 1-13. doi: 10.1016/j.strusafe.2012.01.001.

(23) Altarejos-García, L., Escuder-Bueno, I., \& Morales-Torres, A. (2015). Advances on the Failure Analysis of the DamFoundation Interface of Concrete Dams. Materials, 8(12), 8255-8278. doi: 10.3390/ma8125442

(24) Fullana Montoro, J. (2016). Una contribución al establecimiento del margen de seguridad contra el deslizamiento de presas de gravedad en explotación en función de la información disponible (Tesis doctoral). Universidad Politécnica de Valencia.

(25) Morales-Torres, A., Escuder-Bueno, I., Altarejos-García, L., \& Serrano-Lombillo, A. (2016). Building fragility curves of sliding failure of concrete gravity dams integrating natural and epistemic uncertainties. Engineering Structures, 125, 227-235. doi: 10.1016/j.engstruct.2016.07.006.

(26) ABAQUS 6.14 Documentation, Dassault Systèmes, 2014. 